\title{
Cooperative Project by Self-Bending Continuum Arms
}

\author{
Alaa Al-Ibadi \\ University of Salford \\ Computing, Science and Engineering \\ a.f.a.al-ibadi@edu.salford.ac.uk
}

\author{
Samia Nefti-Meziani \\ University of Salford \\ Computing, Science and Engineering \\ S.Nefti-Meziani@salford.ac.uk
}

\author{
Steve Davis \\ University of Salford \\ Computing, Science and Engineering \\ S.T.Davis@salford.ac.uk
}

\begin{abstract}
Designing a multi-robot system provides numerous advantages for many applications such as low cost, multi-tasking and more efficient group work. However, the rigidity of the robots used in industrial and medical applications increases the probability of injury. Therefore, lots of research is done to increase the safety factor for robot-human interaction. As a result, either separation between the human and robot is suggested, or the force shutdown to the robot system is applied. These solutions might be useful for industrial applications, but it is not for medical applications as a direct interaction between the human and the machine is required. To overcome the rigidity problem, a soft robot arm is presented in this paper. Studying the structure and performance of a contraction pneumatic muscle actuator (PMA) is illustrated, then useful strategies are used to implement a multi PMA continuum arm to increase the performance options for such types of the actuator. Moreover, twin arms are constructed to organise a collaborative project depending on the performance abilities of the proposed arms and end effectors.
\end{abstract}

Keywords-PMA, contraction actuator, self-bending, collaborative system.

\section{INTRODUCTION}

Nowadays, there is a significant interest in robots in industrial, medical and space research. Numerous challenges and difficulties have been found in such robot applications, including the risk of injury, rigidity, position and force control problems, cost and a wide workspace requirement. Many solutions have been introduced to solve these problems; however, a number of difficulties are still being studied.

New controller processes are presented to overcome the position and force control problems of rigid robots, either for single robots or for cooperation groups. Several pieces of research are being done to modify the existing actuators or test new types to make the robot safer for human interaction, which is one of the main challenges in industrial application areas.

Numerous types of variable stiffness actuators (VSA) have been presented during the last few years. Some of them reduced the probability of risk of injury. In spite of their excellent performance, rigidity is the main characteristic of this type of actuator. This led to the invention of sufficiently soft, high stiffness actuators. In recent years there have been a substantial increase in designing, modelling and constructing (biological based) continuum robots $[1,2]$. The PMA has numerous positives over ordinary pneumatic cylinders such as the high force in compression to its weight, low workspace requirement, high flexibility to construct [3, 4], adaptable installation possibilities, minimum consumptions of compressed-air, accessibility of different measurements, low cost and safe for human using [3, 5]. In spite of these exceptional features, the PMA has been considered as appropriate actuators to use behind the electrical and hydraulic actuators.

Despite its distinct positives, the PMA shows extremely nonlinear features[6]. This nonlinearity is caused by the elastic-viscous properties of the inner rubber tube, the compressibility of air, and the structure of the complex behaviour of the PMA outer covering [4]. Furthermore, the hysteresis performance is due to the inner rubber tube, which products different performances of the PMA during different pressurizing condition [3]. This behaviour increases the complexity of system model and its control $[1,7]$.

Numerous researches are done to model the performances of such type of actuators. From these models, the Tondu and Lopez formula [6] and the Chou and Hannaford model [8] are extensively used. Both models are established on the theory of the virtual works of the cylindrical form and the zero walls of the inner rubber tube and the braided sleeve. Their force formulas have been modified many times to reduce the error between the theoretical and exact force values. The force formula has been enhanced by Al-Ibadi, et al. [9] in addition to model the actuator length as a function of different pressurizing conditions. In spite these works, an exact force and position model does not yet exist. As a result, the control strategies have to be modified to overcome the difficulties for the single actuator as well as for the multi PMAs. The arm, which is made from a PMA, is called a continuum arm, provides new robotic behaviours, and offers an infinite number of robot applications.

Walker, et al. [10] design four sections continuum arm, multi-actuators are used in each section to provide the bending and extension performances. Another multi-section soft arm based on PMA has been made and modeled by Godage, et al. [11].

In this paper, a novel design of continuum arm with an end effector is presented and cooperative projects are made to verify its behaviour. Open loop and closed loop control systems are used to control the bending angle. 


\section{THE STRUCTURE OF PMA}

Joseph L. McKibben in the 1950's developed the pneumatic muscle actuator (PMA), which it is simply made from a latex tube bounded by an expandable sleeve [8]. The simplicity of the McKibben artificial actuator structure presented it to use in many applications. Its operational basics are simple: the bounded tension of the inner rubber tube is converted into a vertical pulling force [12]. Different air pressure produces different pulling force values and this axial force is subject to the construction of the PMA Fig.1 shows the general construction of the pneumatic muscle actuator.

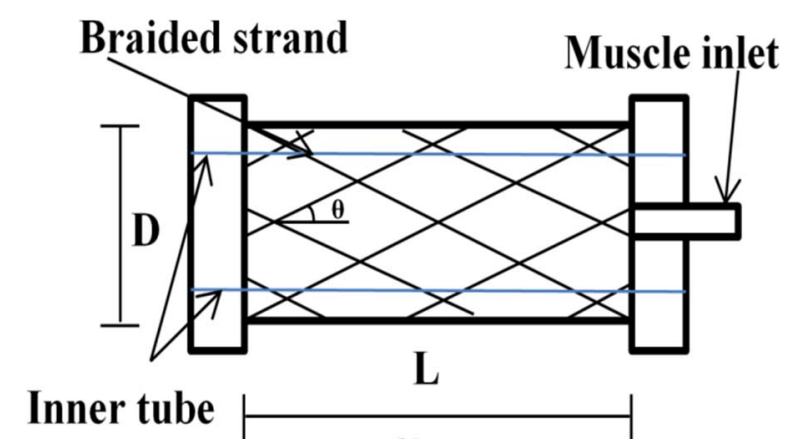

Fig.1. The construction of the PMA

Where the length $\mathrm{L}_{0} \&$ the diameter $\mathrm{D}_{0}$ is measured at zero pressure value and $\theta_{0}$ denote the braided angle, which it is measured between braided strand (b) and the vertical line, its value varies from $0^{\circ}$ to $180^{\circ}$ based on the construction and is a main aspect in the PMA actions.

The length, diameter and the braided angle change with the amount of the air pressure inside the muscle until the diameter reach its maximum value, which it the maximum diameter of the braided sleeve.

The contraction pneumatic muscle actuators act similarly to the biological muscle; the contraction behaviour occurs when the diameter of the inner rubber tube is increasing due to the air pressure and that led to producing a specific amount of pulling force.

\section{OPERATION OF THE PMA}

the principle operation of the contraction pneumatic muscle is fully explained by Kelasidi, et al. [13] in two situations: a) variable air pressure at fixed attached load. b) Varying load and constant air pressure. The diameter of the braided sleeve will increase and as a result the diameter of the PMA rises by incrementing the air pressure while the actuator length will decline to reach the maximum contraction ratio $(\varepsilon)$. Fig.2.10 below describes the operation of the outer sleeve action. And equation 1 gives the contraction ratio expression.

$$
\varepsilon=\frac{L_{0}-L}{L_{0}}
$$

Where $L_{0}$ is the initial actuator length and $L$ is the length of the PMA at pressurise condition.

\section{$x \times x \times x \times x \times$ \\ (a) Before pressurization \\ (b) After pressurization}

Fig.2. The operation behaviour of the braided sleeve

In Fig. 3, n represent the total amount of strand turns for whole the actuator. $\mathrm{n}$ is a constant value for each actuator and it depends on the length and specification of the braided sleeve. The other coefficients such as ( L, D and $\theta$ ) are varied a cause to the amount of air pressure and the construction of the air muscle. In the first operation, as is shown in Fig.3, the actuator is fixed at the upper end and a specific load is attached to its free end. The air pressure slowly increases from zero bar to certain value.

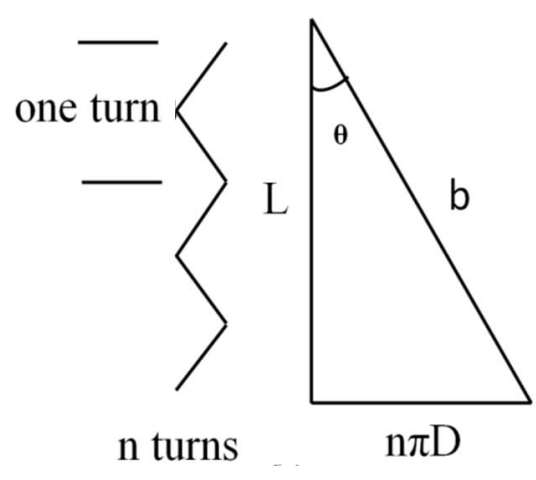

Fig.3. The parameters of the PMA

At a definite pressure value $\mathrm{P}_{1}$, a contracting force will start and lift the fixed load till it reaches the balance point, at this point the contraction force is similar to the mass weight [13].

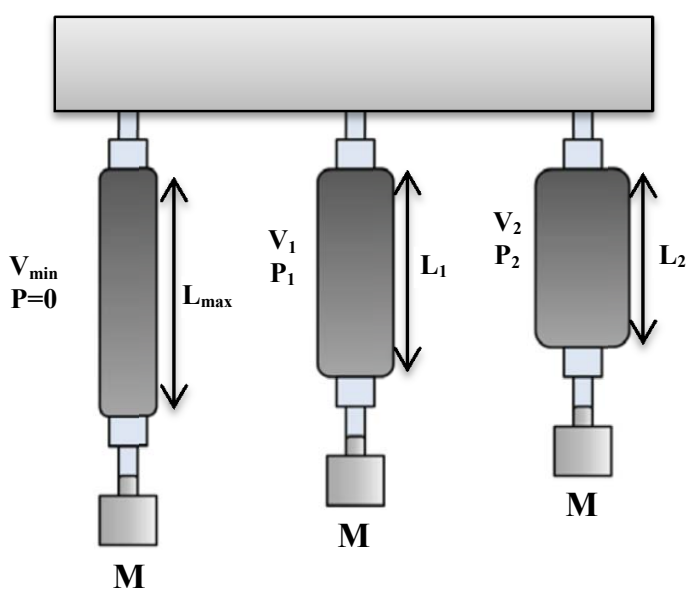

Fig.4. Constant load case of the pneumatic muscle actuator 
The volume of the PMA will rise to $\mathrm{V}_{1}$ and the length reduces to $L_{1}$. Increment the pressure to $P_{2}$ leads to increase the actuator's volume and create more contraction to $\mathrm{L}_{2}$, the air pressure will increase to its maximum value which is subject to the structure of the actuator.

The second action situation is pressurising the PMA at constant air pressure P. Varying the attached load as shown in the Fig. 5 lead to change the behaviour of the PMA. Reducing the load from $M_{1}$ to $M_{2}$ and then to no load will rise the volume and reduce the length of the actuator [13]. Multiple lengths and volumes produce depend on the amount of the air pressure inside the muscle.

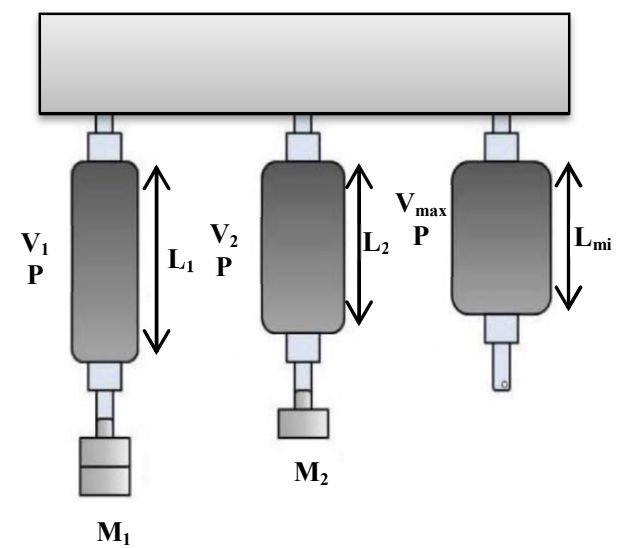

Fig.5. Constant pressure case of the pneumatic muscle actuator

\section{SELF-BENDING CONTRACTION ACTUATOR}

In general, there are two types of PMAs: contraction actuator, which it is fully described above, and the extensor actuator, which behaves in a completely opposite way. The length of the extensor PMA raises when the applied pressure is increased and its diameter decreases.

The contraction performance of the PMA occurs when the braided angle " $\theta$ " is less than $54.7^{\circ}[8,9]$. Moreover, the percentage of contraction differs from one muscle to another but does not exceed 35\% [6]. While, the extension behaviour occur at $\theta>54.7$, the extension ratio is not less than $50 \%$ [14]. To achieve the bending behaviour for the extension PMA, a thread is used to fix one side of the actuator and prevents its extendibility, while the other sides are being free to elongate. The whole muscle will bend toward the thread side. Fig. 6 shows a $30 \mathrm{~cm}$ sewed extensor actuator and its bending at 300 $\mathrm{kPa}$. Moreover, by connecting the extensor actuators in parallel and fixing them together, similar behaviour can be achieved.

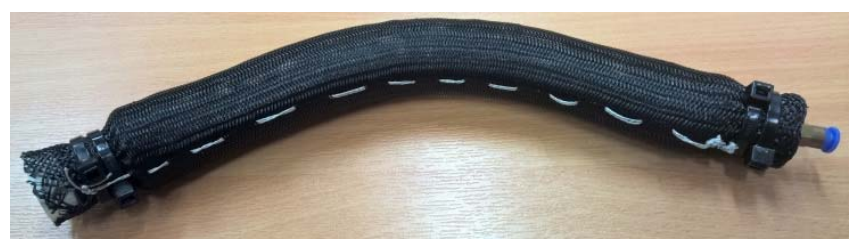

(a)

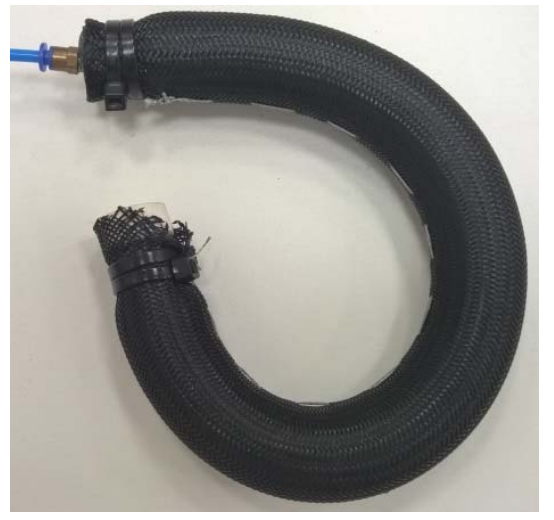

(b)

Fig.6 A $30 \mathrm{~cm}$ extensor PMA (a) one side sewed actuator (b) under $300 \mathrm{kPa}$ air pressure.

Fig.7 illustrates an extensor continuum arm, which is constructed from four actuators of $30 \mathrm{~cm}$ each. The pressure is increased in one of the PMAs in the corner. The arm will then bend into another position, depending on the amount of the air pressure in the muscle and the attached load. The maximum angle at no load is $164.833^{\circ}$, while it is reduced to $116.2^{\circ}$ at 0.5 $\mathrm{kg}$.

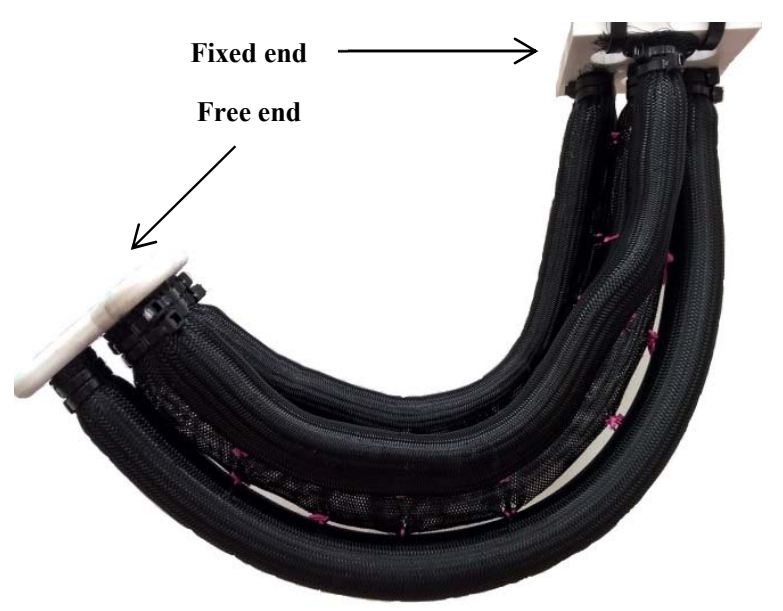

Fig.7 An extensor continuum arm at certain pressure

These two methods are used to reach a bending behaviour of the extensor PMA. On the other hand, the contraction actuator cannot bend by using the thread as in fig. 6 because the actuator length decreases during its operation. Al-Ibadi, et al. [9] built a 4-PMAs arm as shown in fig.8, The bending angle is modelled as a function of air pressure and attached load. The maximum angle without load is about $84.333^{\circ}$ and this angle reduces to $47^{0}$ when the attached load is increased to $0.5 \mathrm{~kg}$. 


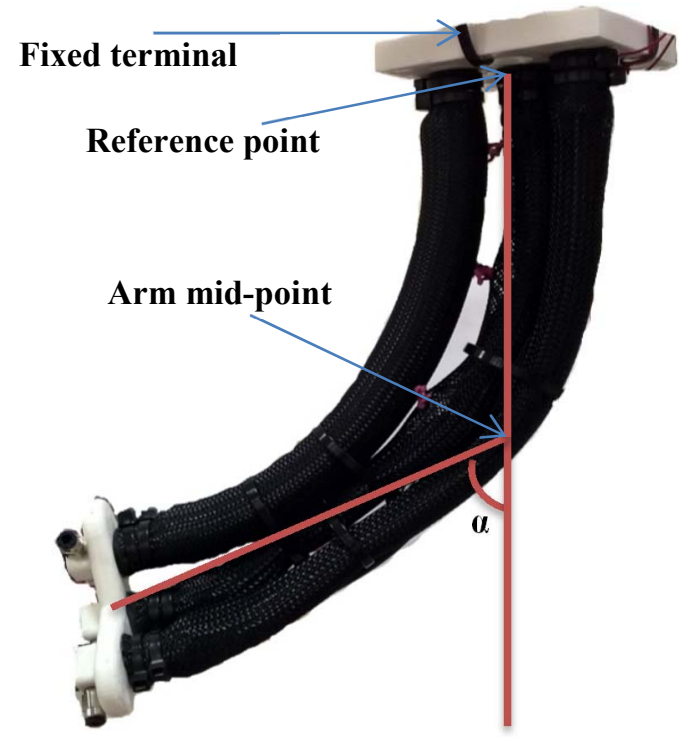

Fig. 8 One PMA pressurized arm with $\alpha$ degree angle.

Increasing the number of actuators increases the complexity of the control system and increase the weight of the robot arm which leads to decrease the weight to force ratio. McMahan, et al. [14] explain that using the principle of the constant-volume creates the bending behaviour of the PMA, where the dimensional adjustment on one side leads to a dimensional modification on another side. This principle has been used above for the single extensor muscle and multi extensor and contractor actuators.

For the contraction actuator, a thin $(2 \mathrm{~mm})$ flexible but incompressible reinforcing rod is made by $3 \mathrm{D}$ printing as shown in Fig. 9.

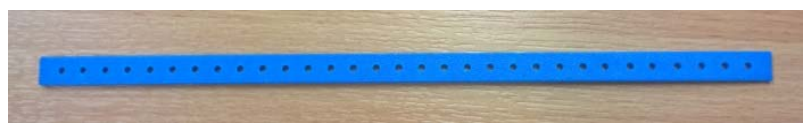

Fig.9. A 3D printed thin rod

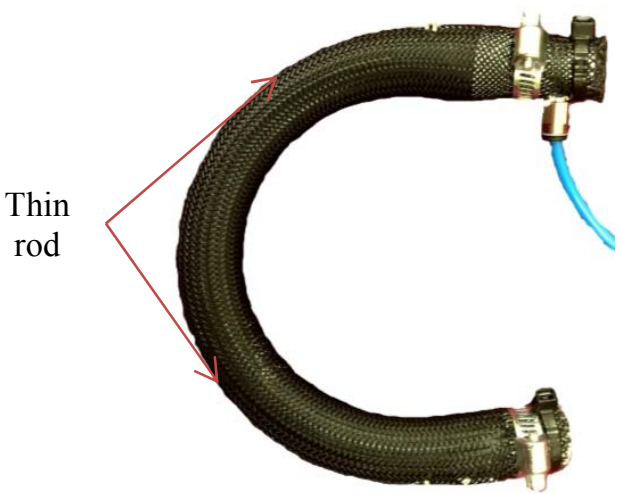

Fig.10. A $30 \mathrm{~cm}$ self-bending contraction PMA
A $30 \mathrm{~cm}$ rod is inserted between the inner rubber tube and the braided sleeve for the $30 \mathrm{~cm}$ contraction actuator and sewed to the sleeve to fix its position. Fig. 10 shows a bent PMA at $300 \mathrm{kPa}$.

A similar technique is used to construct three $10 \mathrm{~cm}$ fingers, which act as a gripper end effector and they are attached to the end of a single actuator. Fig. 11 shows the whole arm.

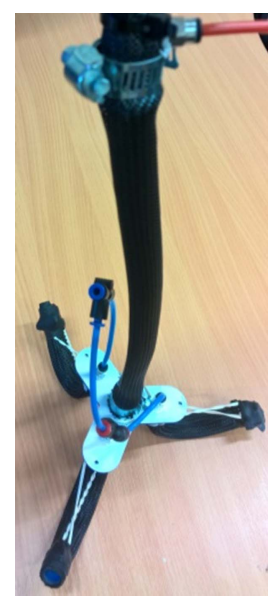

Fig.11. The presented self-bending continuum arm with 3 selfbending fingers

In this proposed arm, the bending angle is higher than the bending angle of the arm shown in fig. 8 . The end effector can be controlled by only controlling the air pressure in a single actuator.

An experiment is done to study the bending angle of the proposed arm at different values of the attached load. Table 1 lists the maximum bending angle at different loads.

Table1. The maximum bending angle at different loads

\begin{tabular}{|c|c|}
\hline Load $(\mathrm{Kg})$ & $\alpha_{\max }($ degree $)$ \\
\hline 0.0 & 213.1 \\
\hline 0.5 & 136.2 \\
\hline 1.0 & 73 \\
\hline 1.5 & 49 \\
\hline 2.0 & 34 \\
\hline
\end{tabular}

From table 1, the bending angle at load $0.5 \mathrm{~kg}$ is $136.2^{0}$, while it is $116.2^{\circ}$ for the 4-PMAs extensor arm in fig. 7 and $47^{\circ}$ for the 4-PMAs contraction arm in fig.8 at similar load values. The payload for the self-bending arm presented is more than the payload for previous arms. Therefore, this arm is more suitable for industrial applications that require efficient bending angles.

Fig. 12 shows the bending angle as a function of air pressure at different load values. 


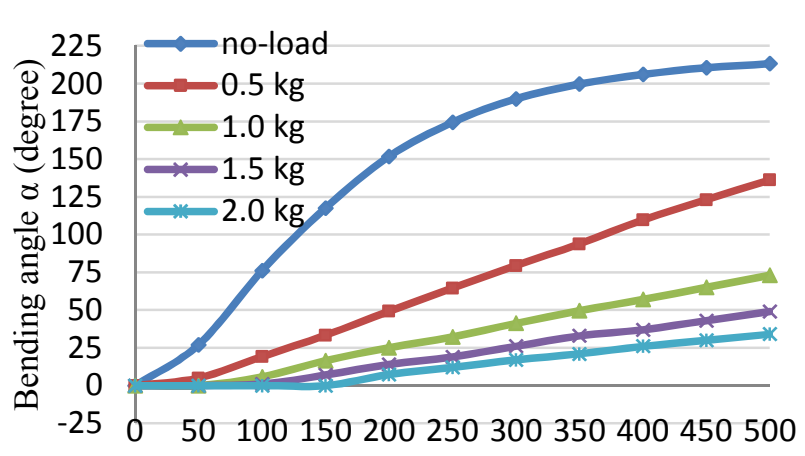

Pressure $\mathrm{kPa}$

Fig.12. Bending angle at different attached load

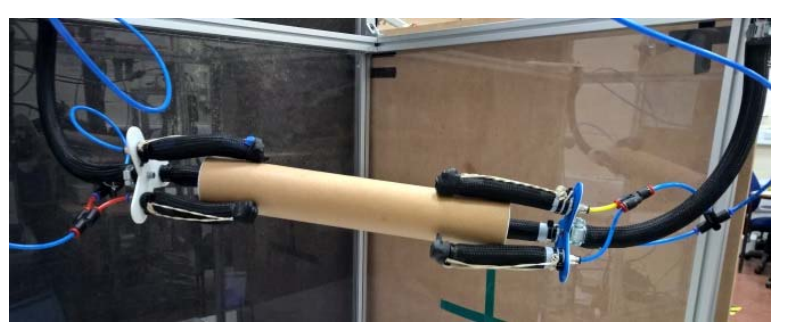

Fig.13. Two similar arms at $90^{\circ}$

\section{COLlabORATIVE SYSTEM}

Similar self-bending arms can make a cooperative project with minimum control system requirements. The first project is made from two similar arms (twins) by applied open loop control to pick up an object then bend to $90^{\circ}$ the second arm then bend to a similar angle and catch the object. After that the two arms return to the vertical mode. Fig. 13 illustrates the arms at $90^{\circ}$. Controlling the air pressure inside the actuator will control the bending angle which is subject to the weight of the object.

To make the twin arms more effective for handling objects of different weights, a closed loop neural network (NN) controller is applied to control the bending angle of the arm. A MPU 6050 sensor has been used to demonstrate the angle of the end effector and adjusting the actuator air pressure by using $(754,3 / 3)$ matrix solenoid valve and an air pressure sensor. The set point of the angle is $90^{\circ}$ to matching between the twin arms.

According to Fig.12, the load to be handled has to be not greater than $0.8 \mathrm{~kg}$ to achieve this collaborative project.

To demonstrate the effectiveness of the controller at different load conditions a square wave set point has been used as illustrated in Fig.14.a and Fig.14.c. From Fig.14.b and Fig.14.d the pressure is increased with an increasing in the attached load. The block diagram of the system is illustrated in Fig. 15.

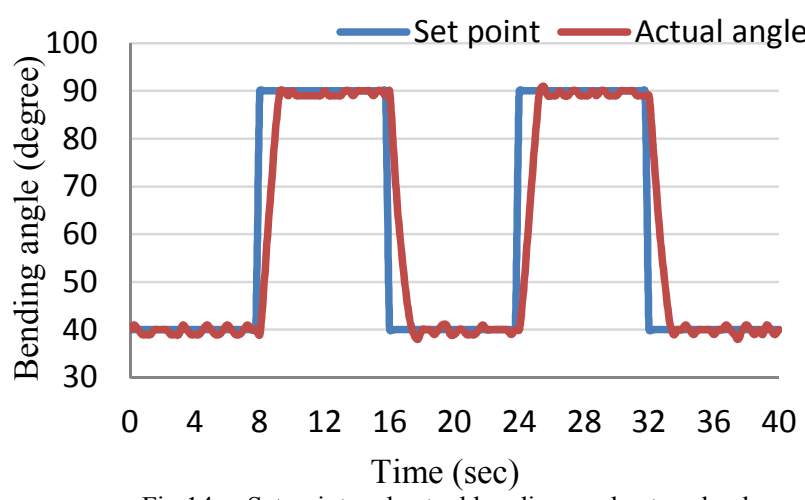

Fig.14.a. Set point and actual bending angle at no-load

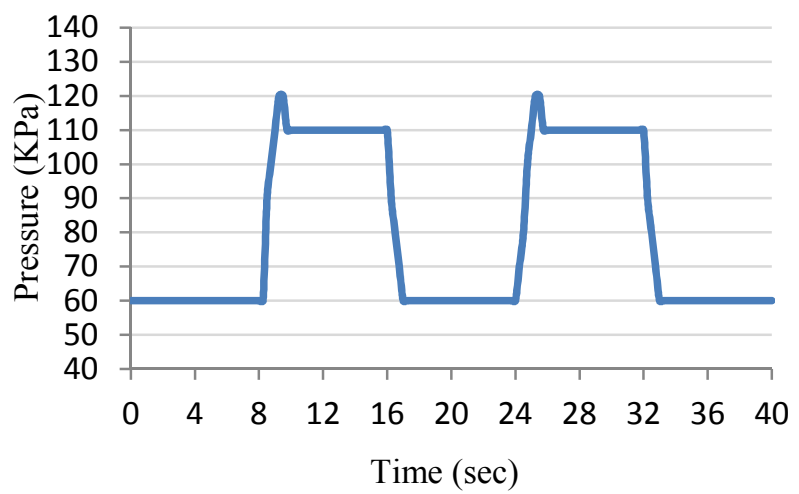

Fig.14.b. The pressure of the bending arm at no-load

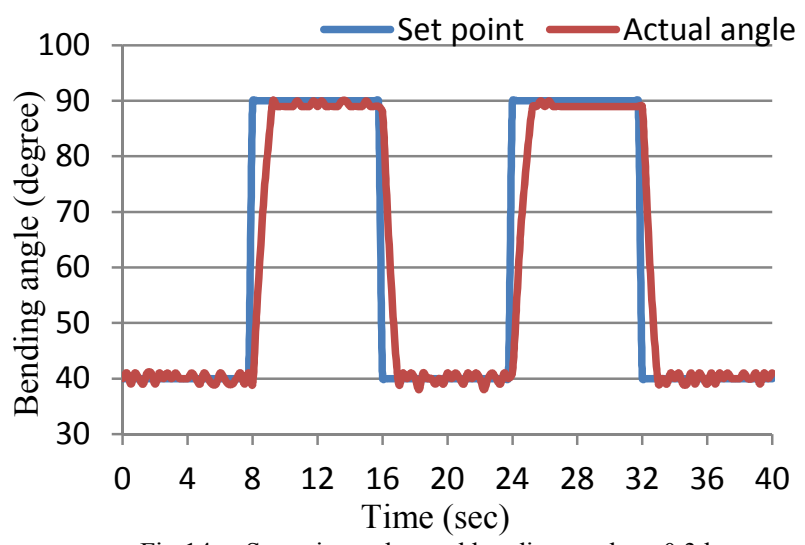

Fig.14.c. Set point and actual bending angle at $0.3 \mathrm{~kg}$

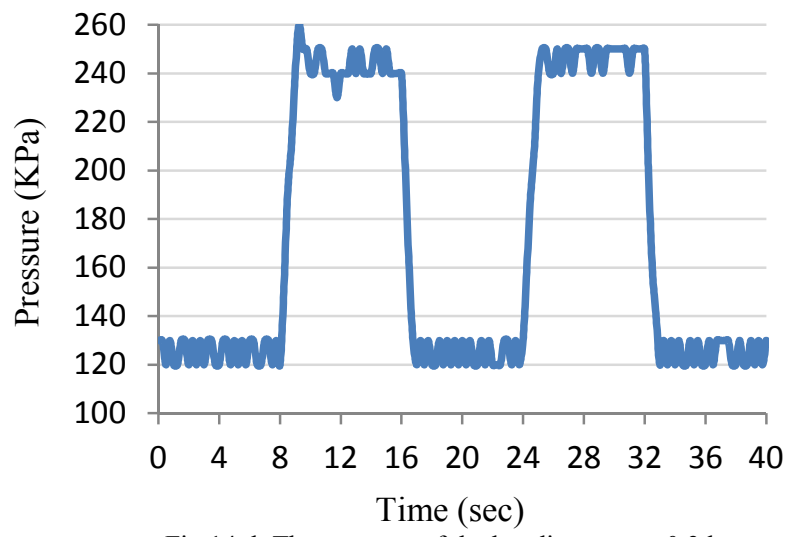

Fig.14.d. The pressure of the bending arm at $0.3 \mathrm{~kg}$ 


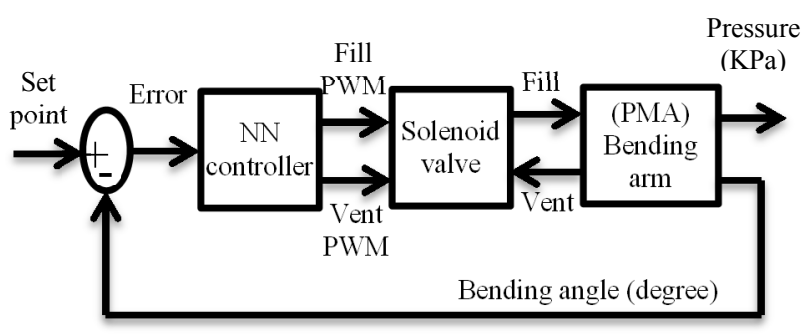

Fig.15. The block diagram of the system

\section{CONCLUSION}

Using soft robots can enhance safety in industrial applications. On the other hand, the robot will play a major role in the medical area. As a result, the humanrobot interaction will be more popular.

In this paper, a single self-bending contractor soft actuator is used to construct two one-direction arms. The arms have been used in multi cooperative projects and the open loop and closed loop controls are applied. In comparison with the previous soft arms, both the bending angle and the bending payload are higher, which makes the presented arm more effectual. Furthermore, the required bending angle can be easily achieved by adjusting the amount of air pressure of single actuator.

As a future work, multi-directional bending arms will be built and the bending payload will be enhanced by such type of actuators. Further sizes of self-bending arms might be constructed which lead to increase the bending payload by increasing the diameter of the actuator. Moreover, controlling the grasping operation depends on the diameter and the weight of the objects.

\section{ACKNOWLEDGMENT}

The authors would like to thank the ministry of higher education/Iraq, University of Basrah, computerengineering department for providing scholarship support to the first author of this paper.

\section{REFERENCES}

[1] A. Bartow, A. Kapadia, and I. Walker, "A novel continuum trunk robot based on contractor muscles," in Proceedings of the 12th WSEAS International Conference on Signal Processing, Robotics, and Automation, 2013, pp. 181-186.

[2] W. McMahan, B. A. Jones, and I. D. Walker, "Design and implementation of a multi-section continuum robot: Air-Octor," in 2005 IEEE/RSJ International Conference on Intelligent Robots and Systems, 2005.(IROS 2005). , 2005, pp. 2578-2585.

[3] B. Mazzolai, L. Margheri, M. Cianchetti, P. Dario, and C. Laschi, "Soft-robotic arm inspired by the octopus: II. From artificial requirements to innovative technological solutions," Bioinspiration \& biomimetics, vol. 7, p. 025005, 2012.

[4] K. K. Aun and H. P. H. Anh, "System modeling identification and control of the two-link pneumatic artificial muscle manipulator optimized with genetic algorithms," in ICCA 2007. IEEE International Conference on Control and Automation. , 2007, pp. 501-506.

[5] I. D. Walker, "Continuous backbone "continuum" robot manipulators," ISRN Robotics, vol. 2013, 2013.

[6] B. Tondu and P. Lopez, "Modeling and control of McKibben artificial muscle robot actuators," Control Systems, IEEE, vol. 20, pp. 15-38, 2000.

[7] R. Kang, D. T. Branson, T. Zheng, E. Guglielmino, and D. G. Caldwell, "Design, modeling and control of a pneumatically actuated manipulator inspired by biological continuum structures," Bioinspiration \& biomimetics, vol. 8, p. 036008, 2013.

[8] C.-P. Chou and B. Hannaford, "Measurement and modeling of McKibben pneumatic artificial muscles," IEEE Transactions on Robotics and Automation., vol. 12, pp. 90-102, 1996.

[9] A. Al-Ibadi, S. Nefti-Meziani, and S. Davis, "Valuable experimental model of contraction pneumatic muscle actuator," in 2016 21st IEEE International Conference on Methods and Models in Automation and Robotics (MMAR), , Poland, 2016, pp. 744-749.

[10] I. D. Walker, D. M. Dawson, T. Flash, F. W. Grasso, R. T. Hanlon, B. Hochner, et al., "Continuum robot arms inspired by cephalopods," in Defense and Security, 2005, pp. 303-314.

[11] I. S. Godage, E. Guglielmino, D. T. Branson, G. A. Medrano-Cerda, and D. G. Caldwell, "Novel modal approach for kinematics of multisection continuum arms," in Intelligent Robots and Systems (IROS), 2011 IEEE/RSJ International Conference on, 2011, pp. 1093-1098.

[12] B. Tondu, "Modelling of the McKibben artificial muscle: A review," Journal of Intelligent Material Systems and Structures, vol. 23, pp. 225-253, 2012.

[13] E. Kelasidi, G. Andrikopoulos, G. Nikolakopoulos, and S. Manesis, "A survey on pneumatic muscle actuators modeling," in 2011 IEEE International Symposium on Industrial Electronics (ISIE), , 2011, pp. 1263-1269.

[14] W. McMahan, V. Chitrakaran, M. Csencsits, D. Dawson, I. D. Walker, B. A. Jones, et al., "Field trials and testing of the OctArm continuum manipulator," in Robotics and Automation, 2006. ICRA 2006. Proceedings 2006 IEEE International Conference on, 2006, pp. 23362341. 